\title{
Resource Management in a Multi-agent System by Means of Reinforcement Learning and Supervised Rule Learning
}

\author{
Bartłomiej Śnieżyński \\ AGH University of Science and Technology, Institute of Computer Science \\ Kraków, Poland \\ Bartlomiej.Sniezynski@agh.edu.pl
}

\begin{abstract}
In this paper two learning methods are presented: reinforcement learning and supervised rule learning. The former is a classical approach to a learning problem in multi-agent systems. The latter is a novel approach, according to the author's knowledge, which has several advantages. Both methods are used for resource management in a multi-agent system. The environment is a Fish Bank game, where agents manage fishing companies. Both learning methods are applied to generate ship allocation strategy. In this article the system architecture and learning processes are described and experimental results comparing the performance of implemented types of agents are presented.
\end{abstract}

Keywords: Machine learning, multi-agent systems, resource management.

\section{Introduction}

Resource allocation is a vital problem in multi-agent systems. Agents try to realize theirs goals in a way that is as good as possible, whereas a cooperation may be necessary to avoid an exhaustion of resources and appearing a crisis situation. One of the main problems in the development of such systems is designing an appropriate strategy for resource allocation.

Applying learning algorithms allows to overcome such problems. One can implement an agent that is not perfect, but improves its performance. This is why machine learning term appears in a context of agent systems for several years. There are many learning methods that can be used to generate knowledge or strategy. Choosing an appropriate one, which fits a given problem, sometimes is a difficult task.

In multi-agent systems the most common technique is reinforcement learning [1]. It allows to generate a strategy for an agent in a situation, when the environment provides some feedback after the agent has acted. Feedback takes the form of a real number representing reward, which depends on the quality of the action executed by the agent in a given situation. The goal of the learning is to maximize estimated reward. 
Symbolic, supervised learning is not so widely used in multi-agent systems. There are many methods belonging to this class that generate knowledge from data. Here a rule induction algorithm is used. It generates a rule-based classifier, which assigns a class to a given example. As an input it needs examples, where the class is assigned by some teacher. In the paper it is shown that it is possible to generate such examples in the case of learning resource allocation. Using this method instead of reinforcement learning has several advantages.

As an environment the Fish Banks game is used [2]. It is a simulation where agents run fishing companies that must decide how much, and where to fish.

In the following sections learning in the multi-agent systems is described, developed system and both learning methods are presented, and experimental results are analyzed.

\section{Learning in Multi-agent Systems}

The problem of learning in multi-agent systems may be considered as a union of research on multi-agent systems and on machine learning. Machine learning focuses mostly on research on an isolated process performed by one intelligent module. The multi-agent approach concerns the systems composed of autonomous elements, called agents, whose actions lead to the realization of given goals. In this context, learning is based on the observation of the influences of activities, performed to achieve the goal by an agent itself or by other agents. Learning may proceed in a traditional - centralized (one learning agent) or decentralized manner. In the second case more than one agent is engaged in the learning process [1].

So far agent systems with learning capabilities were applied in many domains: to train agents playing in RoboCup Challenge [3, adapt user interfaces 4, take part in agent-based computational economics simulations [5], analyze distributed data [6].

\section{System Description}

The Fish Banks game is originally designed for teaching people effective cooperation in using natural resources [7. However; it suits using in multi-agent systems very well [2]8. The game is a dynamic environment providing all necessary resources, action execution procedures, and time flow represented by game rounds. Each round consists of the following steps: ships and money update, ship auctions, trading session, ship orders, ship allocation, fishing, fish number update.

Agents represent players that manage fishing companies. Each company aims at collecting maximum assets expressed by the amount of money deposited at a bank account and the number of ships. The company earns money by fishing at fish banks. The environment provides two fishing areas: coastal and deep-sea. Agents can also keep their ships at the port. The cost of deep-sea fishing is the highest. The cost of staying at the port is the lowest but such ship does not 
catch fish. Initially, it is assumed that the number of fish in both banks is close to the bank's maximal capacity. Therefore, at the beginning of game deep-sea fishing is more profitable.

Usually exploration overcomes birth and after several rounds the number can decrease to zero. It is a standard case of "the tragedy of commons" 9. It is more reasonable to keep ships at the harbor then, therefore companies should change theirs strategies.

\subsection{Agents}

Four types of agents are implemented: reinforcement learning agent, rule learning agent, predicting agent, and random agent. The first one uses learned strategy to allocate ships, the second one uses rules induced from the experience to classify actions and chose the best one, third agent type uses previous fishing results to estimate values of different allocation actions, the last one allocates ships randomly.

All types of agents may observe the following aspects of the environment: arriving of new ships bought from a shipyard, money earned in the last round, ships allocations of all agents, and fishing results for deep sea and inshore area. All types of agents can execute the following two types of actions: order ships, allocate ships.

Order ships action is currently very simple. It is implemented in all types of agents in the same way. At the beginning of the game every agent has 10 ships. Every round, if it has less then 15 ships, there is $50 \%$ chance that it orders two new ships.

Ships allocation is based on the method used in [2]. The allocation action is represented by a triple $(h, d, c)$, where $h$ is the number of ships left in a harbor, $d$ and $c$ are numbers of ships sent to a deep sea, and a coastal area respectively. Agents generate a list of allocation strategies for $h=0 \%, 25 \%, 50 \%, 75 \%$, and $100 \%$ of ships that belong to the agent. The rest of ships $(r)$ is partitioned; for every $h$ the following candidates are generated:

1. All: $(h, 0, r),(h, r, 0)$ - send all remaining ships to a deep sea or coastal area,

2. Check: $(h, 1, r-1),(h, r-1,1)$ - send one ship to a deep sea or coastal area and the rest to the other,

3. Three random actions: $(h, x, r-x)$, where $1 \leq x<r$ is a random number allocate remaining ships in a random way,

4. Equal: $(h, r / 2, r / 2)$ - send equal number of ships to both areas.

The random agent allocates ships using one of the candidates chosen by random. Predicting agent uses the following formula to estimate the value of the action:

$$
v(a)=\operatorname{income}(a)+\eta \operatorname{ecology}(a)
$$

where income $(a)$ represents the prediction of the income under the assumption that in this round fishing results will be the same as in the previous round, ecology $(a)$ represents ecological effects of the action $a$ (the value is low if fishing 


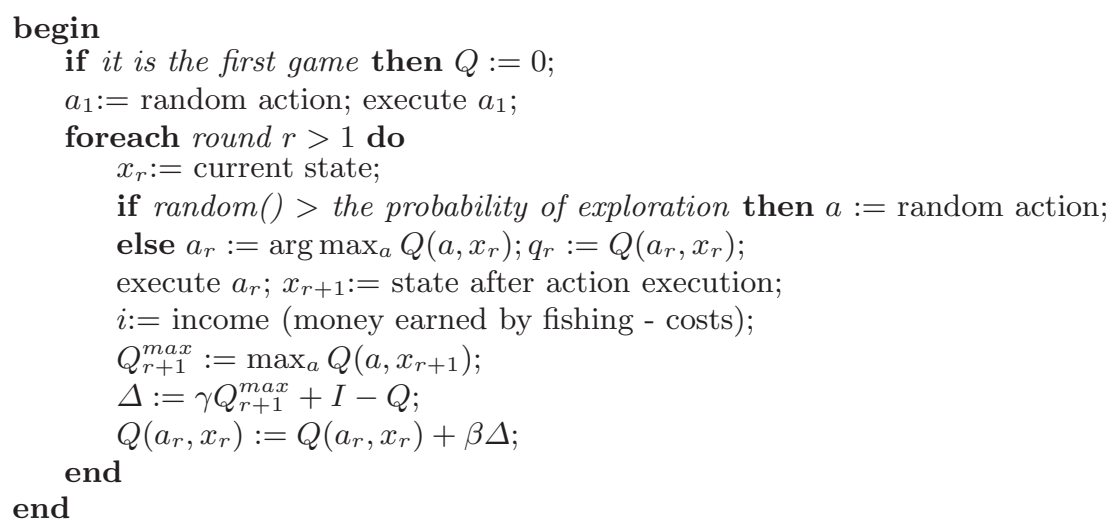

Fig. 1. Algorithm used by the reinforcement learning agent

is performed in the area with low fish population), and $\eta$ represents importance of the ecology factor.

\subsection{Learning Agents}

Reinforcement learning agent chooses action by random in the first round. In the following rounds, action with the highest predicted value $(Q)$ is chosen. $Q$ is a function that estimates value of the action in a given state:

$$
Q: A \times X \rightarrow \Re,
$$

where $A=\{(h, d, c): h, d, c \in\{0 \%, 25 \%, 50 \%, 75 \% 100 \%\}, d+c=1\}$ is a set of all possible ship allocation actions, and $X=\{(d c, c c): d c \in\{1,2, \ldots 25\}, c c \in$ $\{1,2, \ldots, 15\}\}$ is a set of states, which represent catch in both areas in the previous round. As we can see, the set of ship allocation actions is different than one of the other agents. Q-learning algorithm [10] is used to update the $Q$ function after each round (beginning from the second one to have a catch data). The algorithm of the agent is presented in Fig. 1

At the beginning $Q$ is initialized as a constant function 0 . To provide sufficient exploration, in a game number $g$ a random action is chosen with probability $1 / g$ (all actions have the same probability then). Therefore random or the best action (according to $Q$ function) is chosen and executed. Income, which represents feedback from the environment, is calculated, and $Q$ is updated taking into account the reward. The $\gamma \in[0,1]$ parameter represents the importance of the future rewards, $\beta \in(0,1)$ is used to control the speed of change of $Q$.

The rule learning agent also randomizes actions in the first game, but in the following games it chooses action with the highest rating. The action rating is generated using rules, which are stored in the $K B$. Rules allow to classify the allocation as good or bad taking into account allocation parameters and environment parameters (fish catch at the deep sea and at the coastal area in the previous round). The algorithm of the agent is presented in Fig. 2 


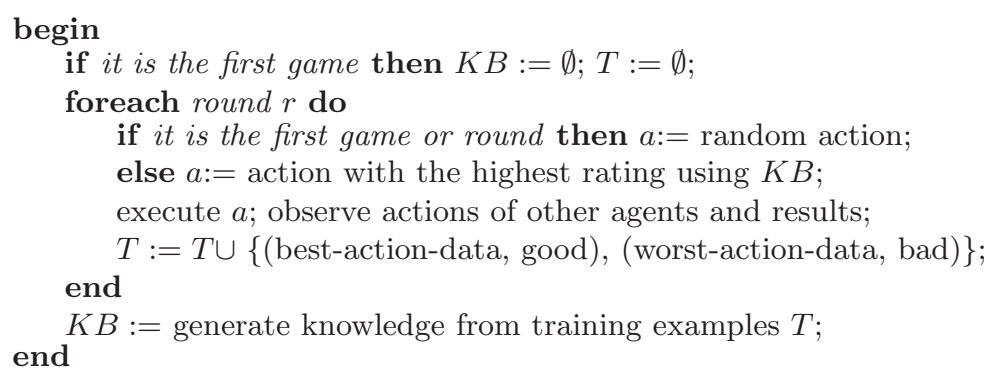

Fig. 2. Algorithm used by the rule learning agent

Every action $a$ gets a value $v$ according to the formula:

$$
v(a)=\alpha \operatorname{good}(a)-\operatorname{bad}(a)
$$

where $\operatorname{good}(a)$ and $\operatorname{bad}(a)$ are numbers of rules, which match the action and current environment parameters, with consequence good and bad, respectively, and $\alpha$ is a weight representing the importance of rules with consequence good.

If there is more then one action with the same value, one occurring earlier in the list is chosen. As a consequence, actions with smaller $h$ are preferred.

Training examples are generated from agents observations. Every round the learning agent stores ship allocations of all agents, and the fish catch in the previous round. The action of an agent with the highest income is classified as good, and the action of an agent with the lowest income is classified as bad. If in some round all agents get the same income, none action is classified, and as a consequence, none of them is used in learning.

At the end of each game the learning agent uses training examples $T$, which were generated during all games played so far, to learn a new classifier $(K B)$, which is used in the next game.

To support rule induction the AQ21 program is used [1]. It is a machine learning software that allows to generate attributional calculus rules for given examples. In standard mode it produces a complete and consistent description of the data, but it can also provide rules that are not complete and/or consistent. The main advantage of this program is that the generated knowledge is easy to interpret for human what makes the experimental results easier to check and can be useful in Fish Bank application to teach people. Of course, other methods of rule (or even classifier) learning can be used.

\subsection{Implementation}

The software used in experiments is written in Prolog, using Prologix compiler [12. Every agent is a separate process. It can be executed on a separate machine. Agents communicate with the environment using Linda blackboard. 
Prologix is an extension of BinProlog that has many powerful knowledge-based extensions (e.g. agent language LOT, Conceptual Graphs and KIF support).

\section{Experimental Results}

Three series of experiments were performed to test how learning influences the agent's performance. Four agents took part in every series. Each series consisted of the sequence of 15 games and it was repeated ten times.

In the first series there were three random agents and one reinforcement learning agent (with $\gamma=1$ and $\beta=0.1$ ). The performance of agents measured as a balance at the end of every game is presented in Fig. 3 ra.

In the second series there were three random agents and one rule learning agent (with weight $\alpha=1$ ). The performance of these agents is presented in Fig. 3.b.

In the third series one learning $(\alpha=1)$, one predicting and two random agents were used. The performance of agents is presented in Fig. 3 c.

In all experiments average balance of both types of learning agents increases with the agent's experience, while the performance of the predicting and random agents decreases slightly (because of the learning agents competition). Reinforcement learning agent was a little bit worse then a rule learning agent, but tuning of its parameters $(\beta, \gamma)$ and taking into account actions of other agents during learning should increase its performance.

Examples of rules learned are presented in Fig. 4. Capital letters represent variables that can be unified with any value. Predicate member checks if its first argument belongs to the list that is a second argument. It is used to represent an internal disjunction (expression of the form $x=v_{1} \vee v_{2} \vee \ldots \vee v_{n}$ ). Remaining predicates represent the catch in the previous round (prevCatchDeep, prevCatchCoastal) and action (harbor, deep_coastal_ratio).

These rules (in the form of clauses) can be interpreted in the following way.

Clause (a): it is a bad decision to keep at a harbor 25, 50, or 75 percent of ships if the previous catch at deep-sea is greater or equal to 16 , and the previous catch at coastal area is 10 .

Clause (b): it is a good decision to send $100 \%$ ships to a deep sea or $75 \%$ to a deep sea and $25 \%$ to a coastal area if previous catch at deep-sea is greater or equal to 18 , and smaller or equal to 21 , and previous catch at coastal area is smaller or equal to 10 .

Experimental results show that the rule learning agent performance increases rapidly at the beginning of the learning process, when generated rules are used instead of a random choice. Next it increases slowly, because new examples do not contain any significant knowledge. The performance stabilizes at the end of the process.

As we can see in Fig. 3-c, the predicting agent performs better then the learning agent. It suggests, that there is a space for improvement of the learning method. Further research is necessary to check if it is possible to learn such a good strategy. 
(a)

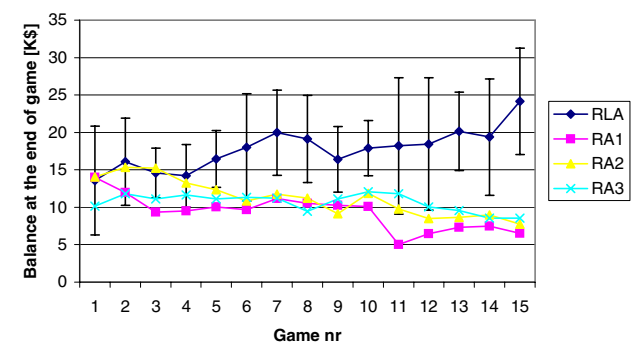

(b)

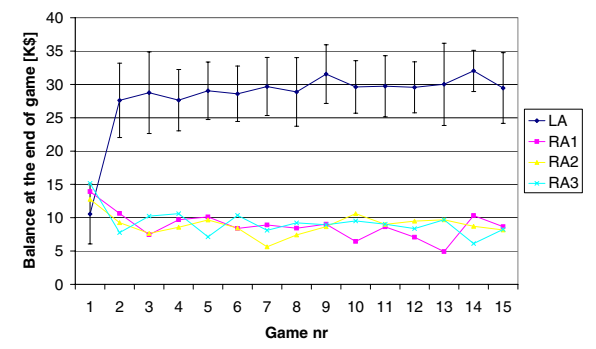

(c)

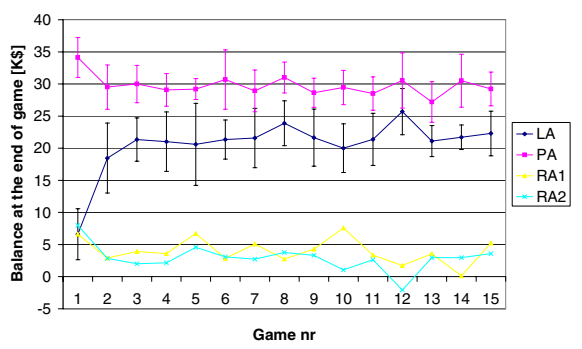

Fig. 3. Comparison of performance of reinforcement learning agent (RLA), rule learning (LA) and other agents using random strategy of ship allocation (RA1, RA2, RA3) or prediction (PA); values for learning and predicting agents are presented with the standard deviation

(a)

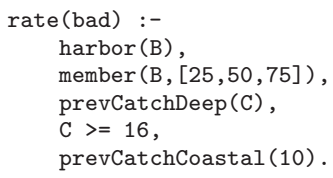

(b)

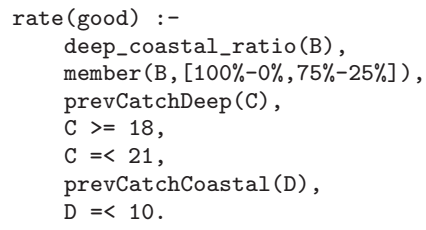

Fig. 4. Examples of rules (in the form of Prolog clauses) learned by the agent

\section{Conclusion and Further Research}

As we can see, both learning algorithms can be applied for learning resource allocation in a multi-agent system. Their performance is much better then a random strategy, but there is still a space for improvement. Both of the techniques have some advantages and disadvantages.

These two methods use different knowledge representation. Reinforcement learning uses the action value function, which is difficult to analyze especially in a case of a large domain. Rules are usually much easier to interpret (unless 
there are too many of them). Therefore, if learned knowledge is analyzed by a human, rule induction seems to be a better choice.

A disadvantage of reinforcement learning is necessity of tuning its parameters $(\gamma, \beta$, and exploration method). The choice has a high impact on the results. What is more, due to necessary exploration, the algorithm's performance is less stable (there is a high variance).

On the other hand, reinforcement learning works well even if the reward is delayed. Additionally, it does not need information about other agents' actions. Hence it is more universal. Rule learning can be modified not to use this data, but it will probably result in slower learning. It will be tested in the future.

Other future works will concern applying other learning algorithms, and cooperation learning. Also applying both methods at the same time for different aspects seems to be an interesting issue.

\section{References}

1. Sen, S., Weiss, G.: Learning in multiagent systems. In Weiss, G., ed.: A Modern Approach to Distributed Artificial Intelligence. The MIT Press (1999)

2. Kozlak, J., Demazeau, Y., Bousquet, F.: Multi-agent system to model the fishbanks game process. In: The First International Workshop of Central and Eastern Europe on Multi-agent Systems (CEEMAS'99), St. Petersburg (1999)

3. H.Kitano, M.Tambe, P.Stone, M.Veloso, S.Coradeschi, Osawa, E., Matsubara, H., Noda, I., Asada, M.: The RoboCup synthetic agent challenge 97. In: International Joint Conference on Artificial Intelligence (IJCAI97), Nagoya, Japan (1997) 24-29

4. Lashkari, Y., Metral, M., Maes, P.: Collaborative interface agents. In: AAAI. (1994) 444-449

5. Tesfatsion, L.: Agent-based computational economics: Growing economies from the bottom up. Artificial Life 8 (1) (2001) 55-82

6. Stolfo, S.J., Prodromidis, A.L., Tselepis, S., Lee, W., Fan, D.W., Chan, P.K.: Jam: Java agents for meta-learning over distributed databases. In: KDD. (1997) 74-81

7. Meadows, D., Iddman, T., Shannon, D.: Fish Banks, LTD: Game Administrator's Manual. Laboratory of Interactive Learning, University of New Hampshire, Durham, USA (1993)

8. Sniezynski, B., Kozlak, J.: Learning in a multi-agent approach to a fish bank game. In: Multi-Agent Systems and Applications IV: Proc. of CEEMAS 2005. Volume 3690 of Lecture Notes in Computer Science. (2005) 568-571

9. Hardin, G.: The tragedy of commons. Science 162 (1968) 1243-1248

10. Watkins, C.J.C.H.: Learning from Delayed Rewards. PhD thesis, King's College, Cambridge (1989)

11. Wojtusiak, J.: AQ21 User's Guide. Reports of the Machine Learning and Inference Laboratory, MLI 04-3. George Mason University, Fairfax, VA (2004)

12. A. Majumdar, P. Tarau, J.S.: Prologix: Users guide. Technical report, VivoMind LLC (2004) 